\title{
MICRO CORIOLIS MASS FLOW SENSOR DRIVEN BY INTEGRATED PZT THIN FILM ACTUATORS
}

\author{
Y. Zeng ${ }^{l}$, J. Groenesteijn ${ }^{1,2}$, D. Alveringh ${ }^{l}$, R.J.A. Steenwelle ${ }^{3}, K^{\prime} . \mathrm{Ma}^{l}$, R.J. Wiegerink ${ }^{l}$, and \\ J.C. Lötters ${ }^{1,2}$ \\ ${ }^{1}$ MESA+ Institute for Nanotechnology, University of Twente, NETHERLANDS \\ ${ }^{2}$ Bronkhorst High-Tech BV, Netherlands \\ ${ }^{3}$ Fraunhofer Project Center at the University of Twente, Netherlands
}

\begin{abstract}
We have realized a micro Coriolis mass flow sensor that is actuated by integrated lead zirconate titanate (PZT) thin film actuators, allowing low voltage, low power actuation compared to current actuation methods. The integrated PZT thin film actuators are deposited on top of silicon-rich silicon nitride ( $\mathrm{SiRN}$ ) fluidic microchannels by pulsed laser deposition (PLD). In this paper we present the fabrication process and first characterization results, including a mass flow measurement with nitrogen flow using a Polytec MSA-400 laser Doppler vibrometer to detect the Coriolis induced motion of the sensor tube. The measurement results confirm that the sensor output is proportional to mass flow up to $14 \mathrm{mg} / \mathrm{min}$.
\end{abstract}

\section{INTRODUCTION}

Integrated micro fluidic systems have generated interest in various fields such as medical [1] and micro chemical technology [2]. Accurate flow measurement of small flows is a very important component in these technologies. Coriolis flow sensing is a preferred choice for flow measurement because of its ability to directly measure mass flow regardless of fluid properties.

Micro Coriolis flow sensors previously reported are driven by either electrostatic force [3] or Lorentz force [4]. However, electrostatic actuation requires high voltage while Lorentz actuation results in relatively large power consumption and Joule heating of the sensor tube and the required strong external magnets cause packaging issues. Switching the driving method to piezo actuation can solve these problems. In this paper, we present the first micro Coriolis flow sensor that is driven by integrated PZT thin film actuators.

\section{THEORY AND DESIGN}

Our micro Coriolis flow sensor consist of a suspended flow channel, as shown in Figure 1. The channel is actuated in swing motion as indicated by the blue arrows. The black dashed line represents the rotation axis of the swing motion with angular velocity $\omega$. Because of the swing mode actuation, a mass flow $\Phi_{\mathrm{m}}$ inside the channel will experience Coriolis forces that induce a twist motion around the axis shown in gray. The Coriolis forces generate a torque around the grey axis given by:

$$
\vec{\tau}=-2 W L\left(\vec{\omega} \times \vec{\Phi}_{m}\right)
$$

where $W$ and $L$ are the width and length of the rectangular tube window.

The resulting twist motion will be superimposed on the swing motion. It will have the same frequency, but because the Coriolis forces are proportional to the angular velocity of the actuation mode there will be 90 degrees phase shift. As a result, instead of measuring the ratio between the vibration amplitudes of the Coriolis (twist) mode and actuation (swing) mode, one can obtain a signal proportional to the mass flow by measuring the phase shift between two points at opposite sides of the twist mode axis.

The PZT thin film actuators are shown in yellow near the suspension of the sensor tube. An applied actuation voltage will make the PZT thin film shrink or expand, bending the tube in the upward or downward direction, respectively.

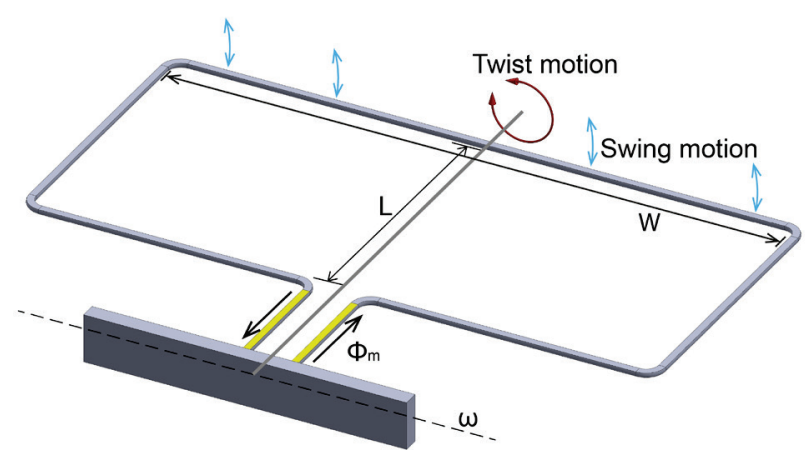

Figure 1: Device operating principle. The yellow parts represent the channel segments with PZT on top. Swing motion is generated by the PZT actuators. Mass flow induces a twist motion due to the Coriolis effect.

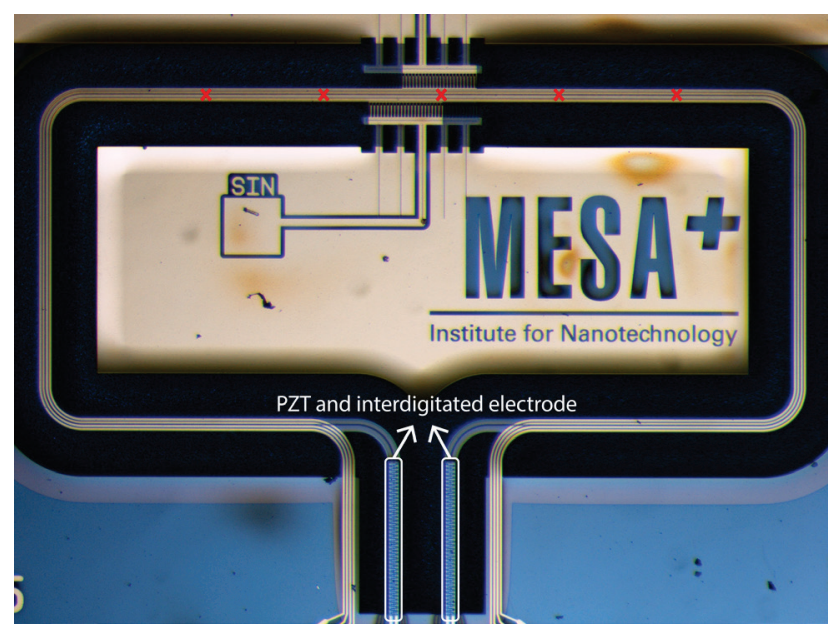

Figure 2: Photograph of sensor chip. The PZT actuators with interdigitated electrodes are located at the suspension of the sensor tube. Thin silicon nitride bridges are used for additional electrical wiring running over the sensor tube. The five red crosses represent the measurement points that are used to measure the motion of the channel using $a$ Polytec MSA-400 laser vibrometer. 
Figure 2 shows a microscope photograph of the sensor chip with the interdigitated actuation electrodes on the sensor channel. Additional gold wiring is running over the sensor tube using thin silicon nitride bridges next to the tube suspension. Figure 3 shows a SEM photograph with a close-up of the tube suspension containing the PZT thin film actuators and the thin silicon nitride bridges containing four parallel gold wires that can be used for capacitive readout and conventional Lorentz force actuation of the sensor. In the current work the additional wiring and capacitive readout structures have not been used.

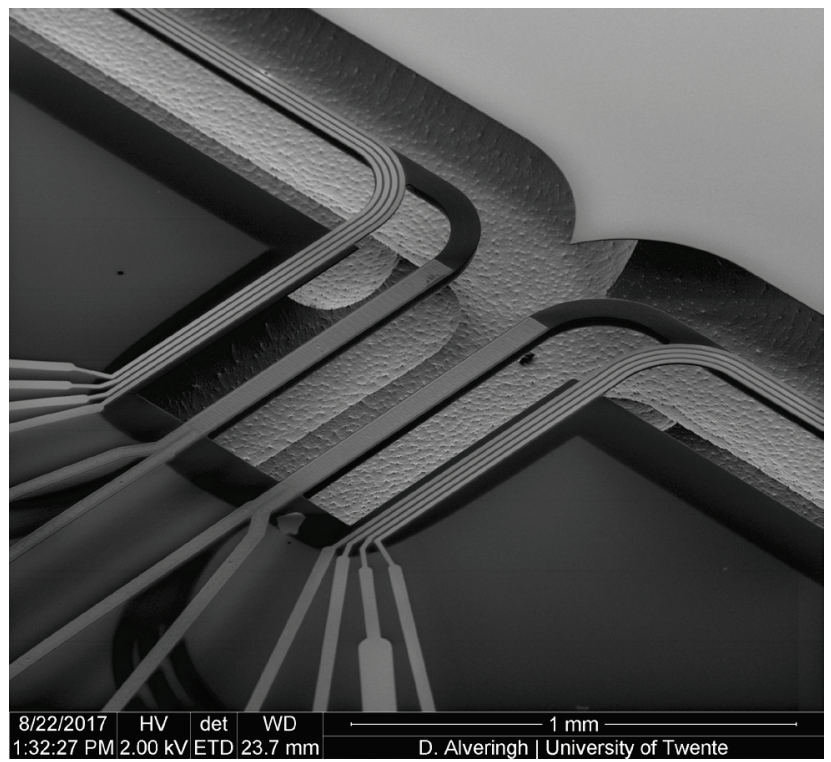

Figure 3: SEM image showing the PZT thin film actuators and the silicon nitride bridges containing additional gold wiring running over the sensor tube.

Figure 4 shows a close-up SEM image of one of the integrated PZT thin film actuators. Interdigitated platinum electrodes are patterned on top of the PZT layer. The electrode width and the distance between the electrodes are both $5 \mu \mathrm{m}$. The entire actuator is $60 \mu \mathrm{m}$ wide and $820 \mu \mathrm{m}$ in length.

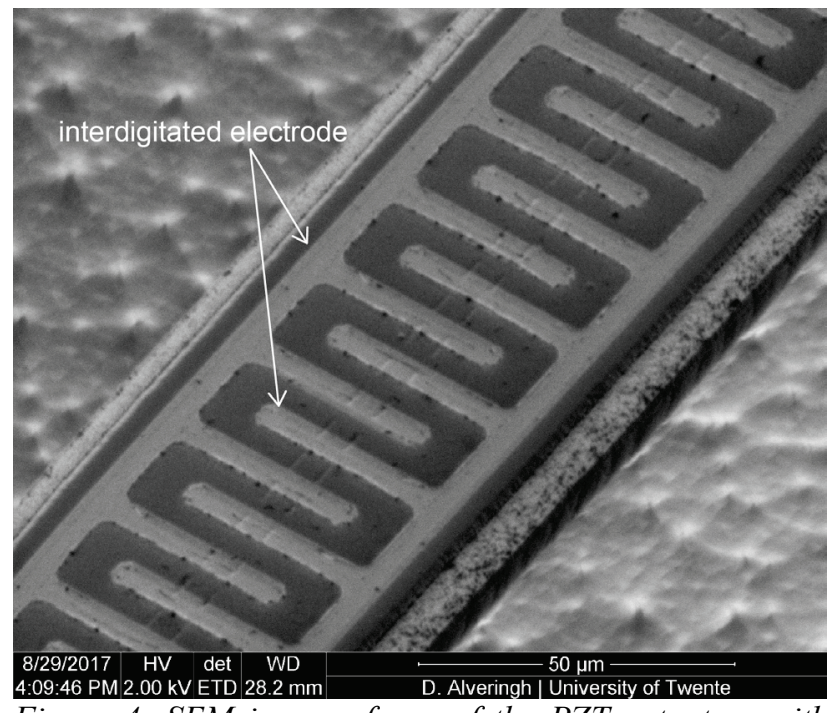

Figure 4: SEM image of one of the PZT actuators with interdigitated platinum electrodes.

\section{FABRICATION}

The fabrication process of the device has been described in [5] with a few additional steps to integrate the PZT actuators. An outline of the fabrication process is shown in Figure 5.

First, a $500 \mathrm{~nm}$ thick layer of SiRN and a $50 \mathrm{~nm}$ thick layer of chromium are deposited on a silicon wafer. The chromium acts as a hard mask for SiRN patterning. After SiRN has been patterned with slits of 5 by $2 \mu \mathrm{m}$, the silicon is isotropically etched through the slits to form the tube shape, see Figure 5(a).

Next, the chromium layer is removed and a layer of silicon dioxide is deposited, as shown in Figure 5(b). Then fluidic inlets are etched from the backside of the wafer using deep reactive ion etching, stopping on the silicon dioxide layer. Subsequently the silicon dioxide layer is selectively removed, resulting in Figure 5(c).

(a)

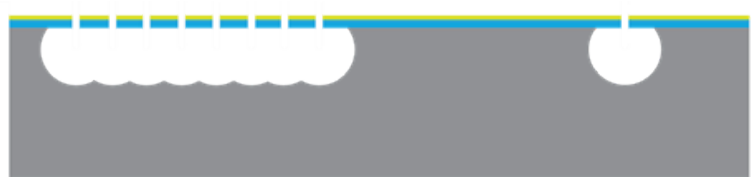

(b)

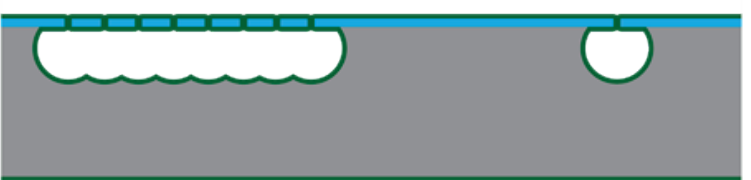

(c)

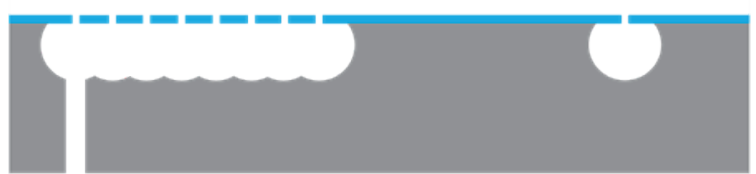

(d)

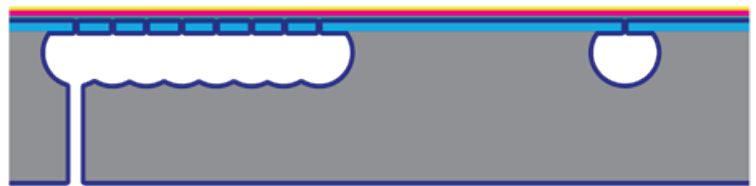

(e)

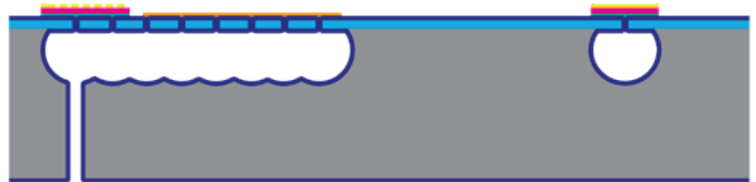

(f)
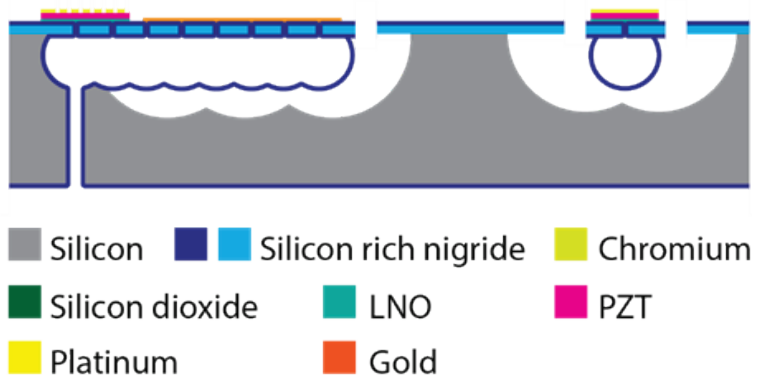

Figure 5: Schematic cross section of the device during the fabrication process. The left side of the figure represents the cross section along the length of the channel. The right side represents the cross section perpendicular to the channel.

Next, another layer of SiRN is deposited by LPCVD 
to form the channel wall and close the slits in the first SiRN layer. Next, the PZT layer is deposited using an $\mathrm{LaNiO}_{3}$ (LNO) seed layer. A Ti/Pt layer is deposited on the top of the PZT layer to form the interdigitated electrodes.

The thicknesses of the LNO/PZT/Ti/Pt layers are $15 \mathrm{~nm} / 1.4 \mu \mathrm{m} / 20 \mathrm{~nm} / 100 \mathrm{~nm}$, respectively. The PLD deposition process has been described in [6]. After deposition of these layers we have the cross section shown in Figure 5(d).

Next, the platinum electrodes and the PZT layer are patterned using ion beam etching and reactive ion etching, respectively. Next, a gold layer with chromium adhesion layer is deposited and patterned to form the additional wiring on the tube and the electrodes for capacitive readout, see Figure 5(e).

Finally, the suspended microfluidic channel is released by etching openings in the SiRN layer followed by isotropic etching of silicon through these openings. The schematic cross section of the final device is shown in Figure 5(f).

\section{FLOW MEASUREMENT SETUP}

The setup we used to measure the flow versus phase shift is shown in figure 6. Nitrogen is supplied from a pressure vessel at 4 bar. The flow is controlled between 0 and $12 \mathrm{ml} / \mathrm{min}$ using a reference flow controller. The sensor is actuated at its resonance frequency by a sinusoidal voltage with an amplitude of $1 \mathrm{~V}$, superimposed on a DC bias voltage of $8 \mathrm{~V}$. The DC bias voltage is needed to avoid changing the poling direction of the PZT thin film.

The twist motion induced by the Coriolis forces is detected using a Polytec MSA-400 laser Doppler vibrometer. The measurement beam of the laser vibrometer is continuously switched between two points on the sensor channel, indicated by "point 1" and "point 2" in Figure 6. The points are chosen symmetrical with respect of the twist mode rotation axis (grey axis in Figure 1). Both points have a relatively large motion due to the swing mode actuation. If there is no flow, the two motions will have the same phase shift with respect to the actuation voltage. The twist motion due to a mass flow inside the channel has a 90 degrees phase shift and therefore results in a phase difference between the motion of the two points. This phase difference is proportional to the amplitude ratio between the twist and swing mode and thus to the mass flow. The two phase shifts $\theta_{1}$ and $\theta_{2}$ with respect to the actuation signal are measured separately by a lock-in amplifier and the difference $\theta_{1}-\theta_{2}$ is calculated.

\section{RESULT AND DISCUSSION}

First the Ferroelectric property of PZT actuators were characterized. The interdigitated electrode on the top of PZT thin film was used in this characterization. The voltage was swept between $-50 \mathrm{~V}$ and $+50 \mathrm{~V}$, corresponding to an applied electric field in the order of $100 \mathrm{kV} / \mathrm{cm}$. Figure 7 shows the measured current (blue curve). By integrating the current the polarization hysteresis loop can be calculated [6] which is also shown in the figure (red curve). It can be concluded that the coercive field $E_{c}$ is around $21 \mathrm{kV} / \mathrm{cm}$. A relatively high leakage current can be observed.
Next, the resonant modes of the device were measured using the Polytec MSA-400 vibrometer. The two PZT thin film actuators were both driven by a $1 \mathrm{~V}$ chirp signal generated by the vibrometer superimposed on a bias voltage of $10 \mathrm{~V}$. The channel was filled with air during this measurement, with both fluid connections open so without any flow through the device. The five measurement points indicated in Figure 2 were scanned to detect the different vibration modes. Figure 8 shows the averaged spectrum.

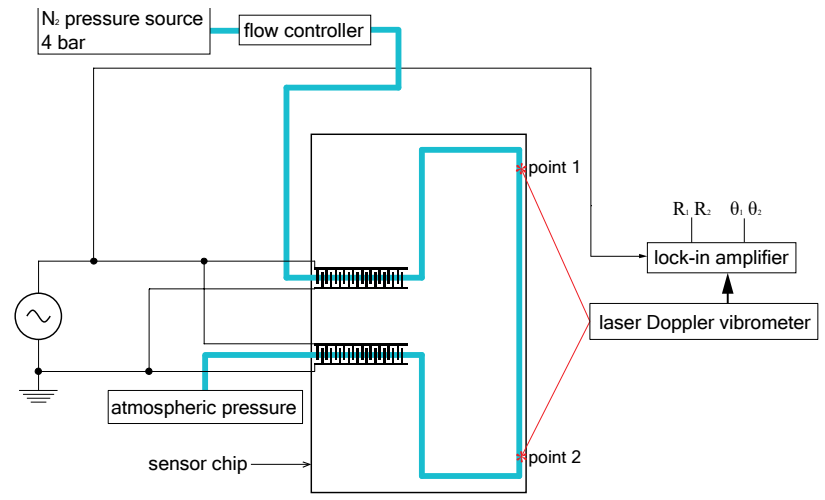

Figure 6: Schematic of the actuation and readout circuit and fluid path. Point 1 and point 2 are located near two corners of the Coriolis channel. $R_{1}$ and $R_{2}, \theta_{1}$ and $\theta_{2}$ represent the amplitude and phase shift of the motion of the two points respectively.

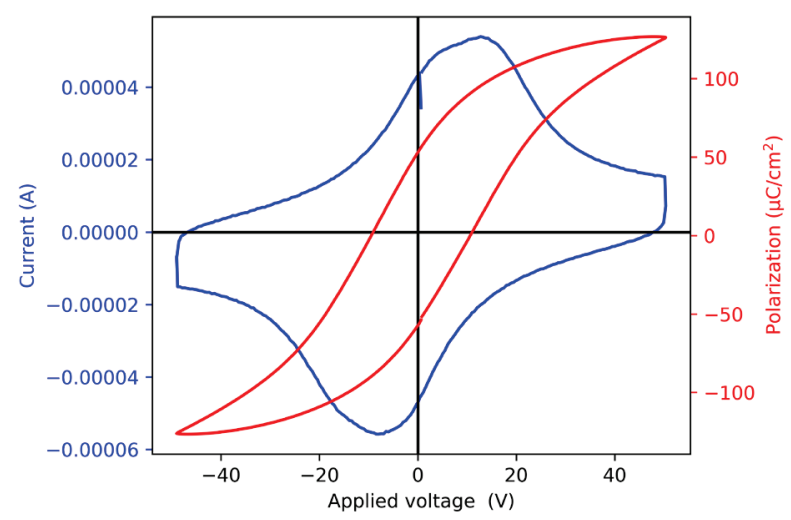

Figure 7: Polarization hysteresis (shown in red) loop and switching current (shown in blue) curve for a PZT thin film actuator. The voltage was swept between $-50 \mathrm{~V}$ and $+50 \mathrm{~V}$ at a frequency of $1 \mathrm{kHz}$.

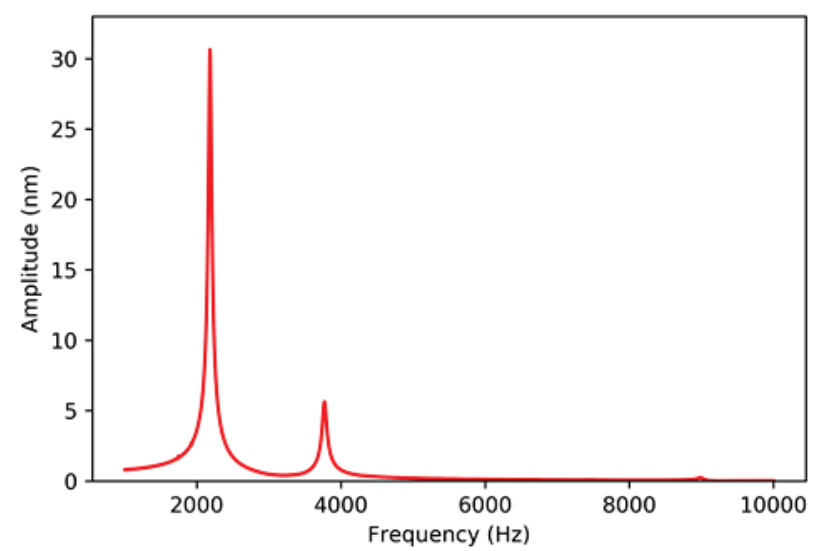

Figure 8: The device resonant spectrum when driven by chirp signal. 
Due to asymmetry induced in fabrication and the PZT deposition process, both the symmetrical and asymmetrical resonance modes are excited. The two peaks in Figure 8 correspond to the swing and twist mode of the device. The resonance frequency of the swing mode is $2184 \mathrm{~Hz}$, with a Q factor of approximately 50 .

Figure 9 shows the vibration amplitude of the five measurement points (Figure 2) when the PZT actuators are driven by a sinusoidal voltage at the resonance frequency as a function of the actuation voltage amplitude. As expected the vibration amplitude increases approximately linear with the actuation voltage. For performing flow measurements an actuation voltage amplitude of $1 \mathrm{~V}$ is chosen, corresponding to a vibration amplitude in the order of $3 \mu \mathrm{m}$.

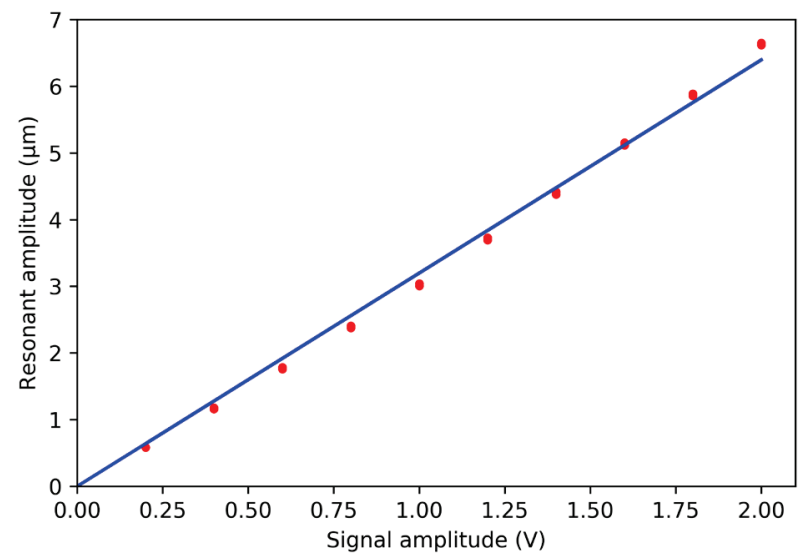

Figure 9: Swing mode vibration amplitude versus driving signal amplitude when the device is driven with a $2184 \mathrm{~Hz}$ sinusoidal signal.

Finally, the measurement setup of Figure 6 was used to perform mass flow measurements. Figure 10 shows the measured phase difference $\theta_{1}-\theta_{2}$ as a function of nitrogen flow between 0 and $12 \mathrm{ml}_{\mathrm{n}} / \mathrm{min}$ (14 $\left.\mathrm{mg} / \mathrm{min}\right)$. As expected, a linear relationship between phase shift and nitrogen flow is observed.

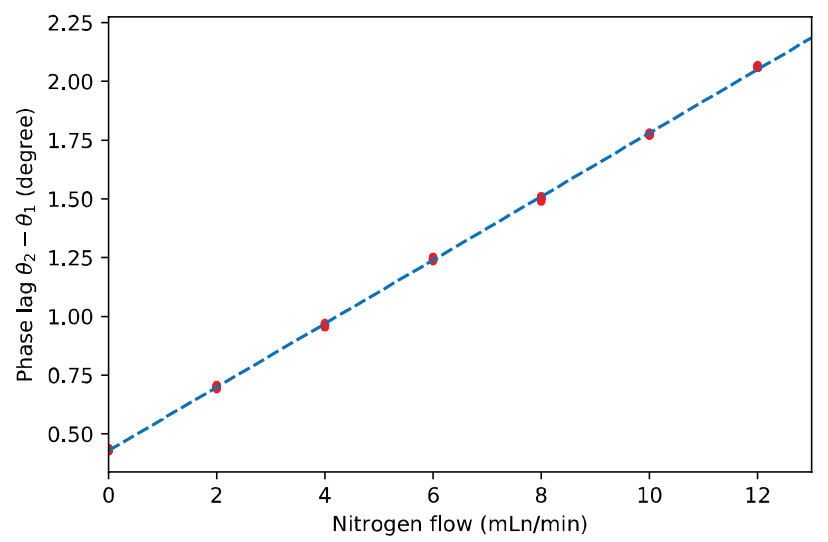

Figure 10: Measured phase shift $\left(\theta_{2}-\theta_{1}\right)$ versus nitrogen flow. The red points are the measured data points. The blue line is a linear fit.

\section{CONCLUSION}

We have successfully fabricated and characterized a micro Coriolis mass flow sensor that was driven by thin film integrated piezoelectric actuators. The device shows a good linear response in experiments using an MSA-400 laser vibrometer to detect the Coriolis motion. The next step is to also integrate the readout on the chip, either a capacitive readout similar to $[4,5]$ or a piezoelectric readout.

\section{ACKNOWLEDGEMENTS}

This work is part of the research programme FLOW+ with project number 15026 which is jointly financed by the Netherlands Organisation for Scientific Research (NWO), Bronkhorst High-Tech, and KROHNE Altometer.

\section{REFERENCES}

[1] D. Figeys and D. Pinto, "Lab-on-a-Chip: A Revolution in Biological and Medical Sciences.," Anal. Chem., vol. 72, no. 9, p. 330 A-335 A, 2000.

[2] K. S. Elvira, X. C. i Solvas, R. C. R. Wootton, and A. J. deMello, "The past, present and potential for microfluidic reactor technology in chemical synthesis," Nat. Chem., vol. 5, no. 11, pp. 905-915, 2013.

[3] P. Enoksson, G. Stemme, and E. Stemme, "A silicon resonant sensor structure for coriolis massflow measurements," J. Microelectromechanical Syst., vol. 6, no. 2, pp. 119-125, 1997.

[4] W. Sparreboom et al., "Compact Mass Flow Meter based on a Micro Coriolis Flow Sensor,' Micromachines, vol. 4, no. 1, pp. 22-33, Mar. 2013.

[5] J. Groenesteijn, M. J. de Boer, J. C. Lötters, and R. J. Wiegerink, “A versatile technology platform for microfluidic handling systems, part I: fabrication and functionalization," Microfluid. Nanofluidics, vol. 21, no. 7, pp. 1-14, 2017.

[6] M. D. Nguyen et al., "Characterization of epitaxial $\mathrm{Pb}(\mathrm{Zr}, \mathrm{Ti}) \mathrm{O} 3$ thin films deposited by pulsed laser deposition on silicon cantilevers," $J$. Micromechanics Microengineering, vol. 20, no. 8, p. $85022,2010$.

\section{CONTACT}

*Y. Zeng, y.zeng-1@utwente.nl 